\title{
NUMERICAL SIMULATION OF IRREGULAR SURFACE ACOUSTIC WAVE EQUATION BASED ON ORTHOGONAL BODY-FITTED GRIDS
}

\author{
LIU, Z. Q. - SUN, J. G. ${ }^{*}$ SUN, H. ${ }^{*}$ - LIU, M. C. ${ }^{*}-$ GAO, Z. H. \\ College for Geoexploration Science and Technology, Jilin University \\ No.938, Ximinzhu Street, Changchun, Jilin Province, 130026, P. R. China \\ (phone: +04-31-8592-3862; fax: +04-31-8592-3862) \\ *Corresponding author \\ e-mail:sun_jg@jlu.edu.cn; sun_hui_jlu@163.com;liu_mch@163.com \\ (Received 22 ${ }^{\text {nd }}$ Jul 2016; accepted $9^{\text {th }}$ Nov 2016)
}

\begin{abstract}
We introduce an orthogonal body-fitted grid generation technique, and employ a high-order finite difference method to simulate the acoustic wave under the orthogonal curvilinear coordinate system. Compared with the rectangular grid, the orthogonal body-fitted grid can describe the rugged topography more accurately and eliminate the false scattering waves caused by ladder-like mesh effectively. Because of the orthogonality of body-fitted gird, the complicated transform and interpolation are not needed, thus improves the calculation efficiency. In this paper, perfectly matched layer (PML) absorbing boundary conditions are adopted under the orthogonal curvilinear coordinate system to eliminate the boundary reflection. The numerical calculation shows that the method can effectively eliminate the false scattering waves and improve the calculation accuracy.
\end{abstract}

Keywords: high-order finite difference; free boundary conditions; PML absorbing boundary conditions; false scattering waves

\section{Introduction}

Seismic wave exploration under the complex surface conditions is a difficult point for the seismic exploration. Dramatic land fluctuations will exert a huge influence on seismic wave propagation, causing various scattered waves and ground roll along the earth's surface. These waves increase the complexity of the wave field and will seriously pollute the seismic records. Besides, current imaging methods have a poor adaptability to irregular topography. As a result, the quality of imaging is not high. Thus, to study the low and characteristic of the seismic wave propagation under irregular surface have an important theoretical and practical significance. The seismic wave numerical simulation is an effective tool to study the propagation of seismic waves within the earth (Sun, 2007). Up to now, various seismic wave numerical simulation methods have been developed for irregular surfaces modeling, including the ray method, the finite element method, the integral equation method and the finite difference method, et al.

Among the methods, the finite difference method is most widely used for its simplicity, high calculation efficiency and easy programming. Conventional seismic simulation through the finite difference method is based on the regular grid under the Cartesian coordinate. However, the ladder-like boundary is unavoidable during the seismic wave simulation under the irregular surface, which might cause the man-made false scattered wave. In order to alleviate the false scattered wave, the fine grid is adopted, which inevitably cause the increase of the calculation memory space and the calculation quantity. In response to the problem, variable mesh and irregular mesh seismic wave simulation methods have been developed. Jastram et al. $(1992,1994)$ put forward the 
vertical variable grid elastic wave numerical simulation method; Hayashi et al. (2001) employed the variable mesh to study the elastic wave under the undulating surface condition; Opršal and Zahradnik (1999) provided the rectangular irregular mesh difference method of the second-order wave equation in the heterogeneous medium; Chu and Wang (2005) present the irregular triangular gird finite difference seismic modeling. In order to completely eliminate the influence of ladder-like mesh approximation, Tessmer et al. (1992) and Hestholm and Ruud (1994) put forward using mapping function to transform the original irregular surface to the regular rectangular grid. Wave equation simulation is conducted on the rectangular grid after transformation. However, the solution of the wave equation by these methods might be unstable when the topographic irregular is huge, so they are just applicable to relatively flat topography. Besides, coordinate transformation is required on the free boundary to meet corresponding boundary conditions. This, on the one hand, increases the calculation quantity; on the other hand, reduces the numerical simulation accuracy. Based on the study of seismic simulation methods for irregular surfaces, this paper introduce the orthogonal body-fitted grid to the irregular surface model's discretization and using the high-order finite difference method to simulate the acoustic wave equation. Since the normal vector of the surface is orthogonal to the surface in our coordinate system, the free boundary condition under the horizontal coordinate can be directly employed. There is no need for complex coordinate transformation and interpolation on the free boundary. In this way, the calculation efficiency is improved.

Establishing the absorbing boundary conditions for the seismic wave numerical simulation is an important issue. In 1977, Clayton present the absorbing boundary conditions based on the paraxial approximation theory. The method shows good absorption effect within certain angle and frequency scope. However, the wave effect of arbitrary angle and frequency is not ideal. Berenger (1994) puts forward the perfectly matched layer (PML) absorbing boundary condition as to electromagnetic wave propagation. The method can fully absorb wave from various directions and of various frequencies without causing any reflection. After that, PML is introduced to seismic wave numerical simulation, and has been successfully applied to the numerical simulation of the acoustic wave, elastic wave and the viscoelastic wave equations. This paper adopts PML absorbing boundary conditions under the orthogonal curvilinear coordinate system to eliminate the man-made boundary reflection.

\section{Theoretical equation}

\section{Body-fitted grid generation method}

Body-fitted grid generation is to transform the irregular region on the physical plane to the regular region on the calculation plane. The corresponding coordinate is called boundary-fitted coordinate or body-fitted coordinate (Sun and Jiang, 2009). The transformation relationship of the 2D body-fitted grid in math can be expressed as:

$$
\left\{\begin{array}{l}
\xi=\xi(x, z) \\
\eta=\eta(x, z)
\end{array}\right.
$$

Where, $(x, z)$ means grid node on the physical plane; $(\xi, \eta)$ means the grid node on the calculation plane( Fig. 1). 


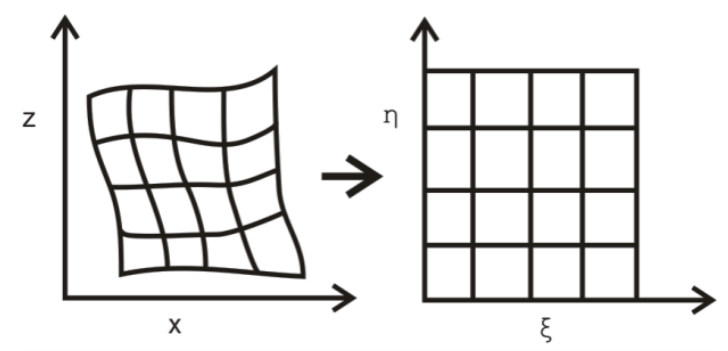

Figure 1. Grid mapping diagram

According to the conformal transformation theory, the real part and the imaginary part of an analytical function are orthogonal and meet the condition of Cauchy-Riemann equation:

$$
\left\{\begin{array}{l}
\nabla^{2} \xi=\xi_{x x}+\xi_{z z}=P \\
\nabla^{2} \eta=\eta_{x x}+\eta_{z z}=Q
\end{array}\right.
$$

Where, $(x, z)$ stands for the grid node on the physical plane; $(\xi, \eta)$ means the grid node on the calculation plane; $P$ and $Q$ are called source items, which are used to adjust the shape and the density of the curvilinear grid on the physical plane. Since the region on the physical plane is irregular, it is quite difficult to calculate the boundary conditions of this equation. Therefore, differential equations are built as follows:

$$
\left\{\begin{array}{l}
\alpha\left(x_{\xi \xi}+\varphi x_{\xi}\right)+\gamma\left(x_{\eta \eta}+\psi x_{\eta}\right)-2 \beta x_{\xi \eta}=0 \\
\alpha\left(z_{\xi \xi}+\varphi z_{\xi}\right)+\gamma\left(z_{\eta \eta}+\psi z_{\eta}\right)-2 \beta z_{\xi \eta}=0
\end{array}\right.
$$

Where,

$$
\left\{\begin{array}{l}
\alpha=x_{\eta}^{2}+z_{\eta}^{2} ; \beta=x_{\xi} x_{\eta}+z_{\xi} z_{\eta} \\
\gamma=z_{\xi}^{2}+z_{\eta}^{2} ; J=x_{\xi} z_{\eta}-x_{\eta} z_{\xi} \\
\varphi=J^{2} P / \alpha ; \psi=J^{2} Q / \gamma
\end{array}\right.
$$

$J$ stands for the Jacobian determinant $J=x_{\xi} z_{\eta}-x_{\eta} z_{\xi}$.

Work out Eq.4 and the coordinate of the curvilinear grid on the physical plane can be obtained, based on which the curvilinear coordinate grid is built.

Since the value of the sources $\varphi, \psi$ have a traction effect on the equivalent $\xi$ line and the equivalent $\eta$ line, respectively, the value of $\varphi, \psi$ can be constantly changed to achieve the expected grid coordinate. Hilgenstock2D2 (Zhang et al., 1997) method use the difference between the practical value and the expected value of the angle between grid line and boundary to implement the orthogonality of the grid. And use the distance between the grid point in the first layer and the boundary to adjust the distance of grid. For example, the correction of the $\varphi, \psi$ on the boundary of $\eta=\eta_{\min }$ is shown below: 


$$
\begin{gathered}
\varphi^{(n+1)}=\varphi^{(n)}-\sigma \tanh \left(\theta_{r}-\theta\right) \\
\psi^{(n+1)}=\psi^{(n)}+\sigma \tanh \left(d_{r}-d\right)
\end{gathered}
$$

Where, $\theta$ stands for the included angle between the $\eta$ grid line and the boundary. $\theta_{r}$ stands for the expected value. Since $\varphi$ has a traction effect on the grid line of $\xi=$ const (the negative value of $\varphi$ can cause $\xi=$ const to move in the decreasing direction; the positive value of $\varphi$ can cause $\xi=$ const to move in the increasing direction), $\varphi$ can be used to traction the grid line of $\xi$ =const, adjust its included angle to be orthogonal to $\eta=$ const ( Fig. 2a). In , $d$ stands for the distance between the grid point of the first layer and the boundary; $d_{r}$ stands for the expected value of the interval (Fig. 2b). Then, when $d_{r}-d>0, d$ shall be increased to make $d$ move close to $d_{r}$. In other words, $\eta=$ const grid line will move to the direction of $\eta$ increase. At the moment, it is necessary to increase $\psi$. On the contrary $\left(d_{r}-d<0\right), \psi$ should be decreased. Therefore, $d_{r}-d$ can be regarded as a correction term to correct the value of $\psi$. Similarly, the correction formula on other boundaries can be built. When the source item value on the boundary is worked out, the source item value of the interior field can be obtained through the interpolation value on the boundary. In this paper, the linear interpolation equation is adopted to work out the source item of the internal grid point. The interpolation value equation is shown below:

$$
\begin{aligned}
& \varphi=\varphi_{u p}+\left(\varphi_{\text {down }}-\varphi_{u p}\right) \frac{\eta-\eta_{0}}{\eta_{n}-\eta_{0}} \\
& \psi=\psi_{u p}+\left(\psi_{\text {down }}-\psi_{u p}\right) \frac{\eta-\eta_{0}}{\eta_{n}-\eta_{0}}
\end{aligned}
$$

Where, $\varphi_{u p}, \psi_{u p}$ stand for the source items on the top boundary of the model; $\varphi_{\text {down }}, \psi_{\text {down }}$ stand for the vertical coordinate of the mesh node on the bottom boundary of the model; $\eta$ stands for the vertical coordinate of the mesh node; $\eta_{0}$ stands for the vertical coordinate of the minimum mesh node; $\eta_{n}$ stands for the vertical coordinate of the maximum mesh node.

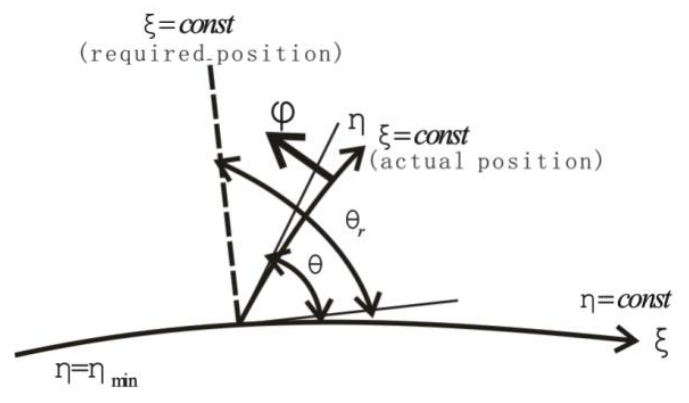

(a)

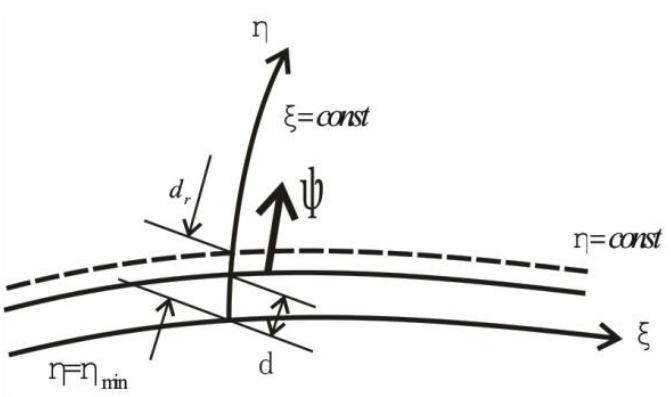

(b)

Figure 2. (a) $\varphi$ to stretch the grid lines $\xi=$ const $;(b) \psi$ to stretch the grid lines $\eta=$ const 


\section{High-order finite difference scheme of acoustic wave equation in the orthogonal curvilinear coordinate}

According to the chain rule, the acoustic wave equation in the orthogonal curvilinear coordinate $(\xi, \eta)$ is as follows:

$$
\left(C_{1}^{2}+C_{2}^{2}\right) \frac{\partial^{2} P}{\partial \xi^{2}}+\left(C_{3}^{2}+C_{4}^{2}\right) \frac{\partial^{2} P}{\partial \eta^{2}}+\left(C_{5}+C_{6}\right) \frac{\partial P}{\partial \xi}+\left(C_{7}+C_{8}\right) \frac{\partial P}{\partial \eta}=\frac{1}{v^{2}} \frac{\partial^{2} P}{\partial t^{2}}
$$

Where,

$$
\begin{aligned}
& C_{1} \equiv \frac{\partial \xi}{\partial x}, C_{2} \equiv \frac{\partial \xi}{\partial z}, C_{3} \equiv \frac{\partial \eta}{\partial x}, C_{4} \equiv \frac{\partial \eta}{\partial z} \\
& C_{5} \equiv \frac{\partial^{2} \xi}{\partial x^{2}}, C_{6} \equiv \frac{\partial^{2} \xi}{\partial z^{2}}, C_{7} \equiv \frac{\partial^{2} \eta}{\partial x^{2}}, C_{8} \equiv \frac{\partial^{2} \eta}{\partial z^{2}}
\end{aligned}
$$

In Eq.7, $P$ stands for the pressure; $v$ stands for the wave spreading speed; $t$ stands for time.

Using time second-order and space eighth-order finite difference scheme to discretize the derivative equation Eq.7, so:

$$
\begin{aligned}
P_{i, j}^{k+1} & =2 P_{i, j}^{k}-P_{i, j}^{k-1}+\frac{\Delta t^{2}}{\Delta \xi^{2}}\left(C_{1}^{2}+C_{2}^{2}\right)\left[a_{0} P_{i, j}^{k}+\sum_{m=1}^{4}\left(P_{i+m, j}^{k}+P_{i-m, j}^{k}\right)\right] \\
+ & \frac{\Delta t^{2}}{\Delta \eta^{2}}\left(C_{3}^{2}+C_{4}^{2}\right)\left[a_{0} P_{i, j}^{k}+\sum_{m=1}^{4}\left(P_{i, j+m}^{k}+P_{i, j-m}^{k}\right)\right] \\
+ & \frac{\Delta t^{2}}{\Delta \xi}\left(C_{5}+C_{6}\right) \sum_{m=1}^{4} b_{m}\left(P_{i+m, j}^{k}-P_{i-m, j}^{k}\right)+\frac{\Delta t^{2}}{\Delta \eta}\left(C_{7}+C_{8}\right) \sum_{m=1}^{4} b_{m}\left(P_{i, j+m}^{k}-P_{i, j-m}^{k}\right)
\end{aligned}
$$

Where,

$$
\begin{aligned}
& a_{0}=-2.8472, a_{1}=1.6000, a_{2}=-0.2000, a_{3}=0.0254, a_{4}=-0.0018 \\
& b_{1}=0.8000, b_{2}=-0.2000, b_{3}=0.0381, b_{4}=-0.0036
\end{aligned}
$$

Below is a deduction of how to solve $C_{1}, C_{2}, C_{3}, C_{4}, C_{5}, C_{6}, C_{7}, C_{8}$. According to the coordinate transformation theory, there exists the following coordinate system transformation relationship:

$$
\xi_{x}=\frac{z_{\eta}}{J}, \xi_{z}=-\frac{x_{\eta}}{J}, \eta_{x}=-\frac{z_{\xi}}{J}, \eta_{z}=\frac{x_{\xi}}{J}
$$

Where, $J$ stands for the Jacobian determinant, $J=x_{\xi} z_{\eta}-x_{\eta} z_{\xi}$

Through Eq.10 and the Jacobian determinant, the following equations can be obtained: 


$$
\begin{aligned}
& \frac{\partial\left(\xi_{x}\right)}{\partial \xi}=\frac{z_{\xi \eta} J-z_{\eta} J_{\xi}}{J^{2}} ; \frac{\partial\left(\xi_{x}\right)}{\partial \eta}=\frac{z_{\eta \eta} J-z_{\eta} J_{\eta}}{J^{2}} \\
& \frac{\partial\left(\eta_{x}\right)}{\partial \xi}=-\frac{z_{\xi \xi} J-z_{\xi} J_{\xi}}{J^{2}} ; \frac{\partial\left(\eta_{x}\right)}{\partial \eta}=-\frac{z_{\xi \eta} J-z_{\xi} J_{\eta}}{J^{2}} \\
& \frac{\partial\left(\xi_{y}\right)}{\partial \xi}=-\frac{x_{\xi \eta} J-x_{\eta} J_{\xi}}{J^{2}} ; \frac{\partial\left(\xi_{y}\right)}{\partial \eta}=-\frac{x_{\eta \eta} J-x_{\eta} J_{\eta}}{J^{2}} \\
& \frac{\partial\left(\eta_{y}\right)}{\partial \xi}=\frac{x_{\xi \xi} J-x_{\xi} J_{\xi}}{J^{2}} ; \frac{\partial\left(\eta_{y}\right)}{\partial \eta}=\frac{x_{\xi \eta} J-x_{\xi} J_{\eta}}{J^{2}} \\
& J_{\xi}=x_{\xi \xi} z_{\eta}+x_{\xi} z_{\xi \eta}-x_{\xi \eta} z_{\xi}-x_{\eta} z_{\xi \xi} \\
& J_{\eta}=x_{\xi \eta} z_{\eta}+x_{\xi} z_{\eta \eta}-x_{\eta \eta} z_{\xi}-x_{\eta} z_{\xi \eta}
\end{aligned}
$$

Assuming that $A=\xi_{x}^{2}+\xi_{z}^{2} ; B=\eta_{x}^{2}+\eta_{z}^{2}$. According to Eq.11 and Eq.12, the following equation can be deducted:

$$
\begin{aligned}
& \xi_{x x}+\xi_{z z}=A\left(B \frac{\partial x}{\partial \eta} \frac{\partial^{2} x}{\partial \xi \partial \eta}+B \frac{\partial z}{\partial \eta} \frac{\partial^{2} z}{\partial \xi \partial \eta}-A \frac{\partial x}{\partial \xi} \frac{\partial^{2} x}{\partial \xi^{2}}-A \frac{\partial z}{\partial \xi} \frac{\partial^{2} z}{\partial \xi^{2}}\right) \\
& \eta_{x x}+\eta_{z z}=B\left(A \frac{\partial x}{\partial \xi} \frac{\partial^{2} x}{\partial \xi \partial \eta}+A \frac{\partial z}{\partial \xi} \frac{\partial^{2} z}{\partial \xi \partial \eta}-A \frac{\partial x}{\partial \eta} \frac{\partial^{2} x}{\partial \eta^{2}}-A \frac{\partial z}{\partial \eta} \frac{\partial^{2} z}{\partial \eta^{2}}\right)
\end{aligned}
$$

Use the difference of the coordinate data to replace the differential of Eq.10and Eq.13 can obtain coefficients $C_{1}, C_{2}, C_{3}, C_{4}, C_{5}, C_{6}, C_{7}, C_{8}$.

\section{Free boundary conditions in the orthogonal curvilinear coordinate system}

According to the elastic wave theory, the free surface should meet the condition that its normal stress is zero, namely

$$
T=\tau \cdot \vec{n}=0
$$

Where, $T$ stands for the stress; $\vec{n}$ stands for the exterior normal vector of the free boundary; $\tau$ stands for the tensor of stress, which can be expressed as below in the curvilinear coordinate system:

$$
\tau=\left[\begin{array}{ll}
\tau_{\xi \xi} & \tau_{\xi \eta} \\
\tau_{\eta \xi} & \tau_{\eta \eta}
\end{array}\right]
$$

After using the orthogonal body-fitted grid to the grid generation of the model, every mesh point on the free surface meets the condition of orthogonality. In other words, the outer normal vector $\vec{n}$, coincide with the coordinate line $\eta$. Then, the free boundary condition of Eq. 15 can be directly expressed as below: 


$$
\left\{\begin{array}{l}
\tau_{\eta \eta}=0 \\
\tau_{\xi \eta}=0
\end{array}\right.
$$

Since the shear stress in the acoustic medium is equal to zero and, the pressure difference $P$ is equal to the negative normal stress, the free boundary condition of the acoustic wave equation can be expressed as below:

$$
\left.P\right|_{\eta=0}=0
$$

\section{PML absorbing conditions in the orthogonal curvilinear coordinate system}

Do the coordinate stretching transform to Eq.7 :

$$
\left\{\begin{array}{l}
\xi=\xi-\frac{i}{\omega} \int_{0}^{\xi} d_{\xi}(s) d s \\
\eta=\eta-\frac{i}{\omega} \int_{0}^{\eta} d_{\eta}(s) d s
\end{array}\right.
$$

By Eq.18:

$$
\left\{\begin{array}{l}
\frac{\partial}{\partial \xi}=\frac{i \omega}{i \omega+d_{\xi}} \frac{\partial}{\partial \xi} \\
\frac{\partial}{\partial \eta}=\frac{i \omega}{i \omega+d_{\eta}} \frac{\partial}{\partial \eta}
\end{array}\right.
$$

Transform Eq.7 to the frequency domain, and use $\xi$ and $\eta$ to replace $\xi$ and $\eta$ :

$$
\left(C_{1}^{2}+C_{2}^{2}\right) \frac{\partial^{2} P}{\partial \xi^{2}}+\left(C_{3}^{2}+C_{4}^{2}\right) \frac{\partial^{2} P}{\partial \eta^{2}}+\left(C_{5}+C_{6}\right) \frac{\partial P}{\partial \xi}+\left(C_{7}+C_{8}\right) \frac{\partial P}{\partial \eta}=\frac{-\omega^{2}}{v^{2}} P
$$

Put Eq.19 into Eq.20, then:

$$
\begin{aligned}
\frac{1}{v^{2}} P & =\left(C_{1}^{2}+C_{2}^{2}\right)\left[\frac{1}{\left(i \omega+d_{\xi}\right)^{2}} \frac{\partial^{2} P}{\partial \xi^{2}}-\frac{1}{\left(i \omega+d_{\xi}\right)^{3}} \frac{\partial d_{\xi}}{\partial \xi} \frac{\partial P}{\partial \xi}\right] \\
& +\left(C_{3}^{2}+C_{4}^{2}\right)\left[\frac{1}{\left(i \omega+d_{\eta}\right)^{2}} \frac{\partial^{2} P}{\partial \eta^{2}}-\frac{1}{\left(i \omega+d_{\eta}\right)^{3}} \frac{\partial d_{\eta}}{\partial \eta} \frac{\partial P}{\partial \eta}\right] \\
& +\left(C_{5}+C_{6}\right) \frac{1}{i \omega\left(i \omega+d_{\xi}\right)} \frac{\partial P}{\partial \xi}+\left(C_{7}+C_{8}\right) \frac{1}{i \omega\left(i \omega+d_{\eta}\right)} \frac{\partial P}{\partial \eta}
\end{aligned}
$$

Assuming that $P=P_{1}+P_{2}+P_{3}+P_{4}$. Then, Eq.21 can be split into the following four equations: 


$$
\left\{\begin{array}{l}
\frac{1}{v^{2}} P_{1}=\left(C_{1}^{2}+C_{2}^{2}\right)\left[\frac{1}{\left(i \omega+d_{\xi}\right)^{2}} \frac{\partial^{2} P}{\partial \xi^{2}}-\frac{1}{\left(i \omega+d_{\xi}\right)^{3}} \frac{\partial d_{\xi}}{\partial \xi} \frac{\partial P}{\partial \xi}\right] \\
\frac{1}{v^{2}} P_{2}=\left(C_{3}^{2}+C_{4}^{2}\right)\left[\frac{1}{\left(i \omega+d_{\eta}\right)^{2}} \frac{\partial^{2} P}{\partial \eta^{2}}-\frac{1}{\left(i \omega+d_{\eta}\right)^{3}} \frac{\partial d_{\eta}}{\partial \eta} \frac{\partial P}{\partial \eta}\right] \\
\frac{1}{v^{2}} P_{3}=\left(C_{5}+C_{6}\right) \frac{1}{i \omega\left(i \omega+d_{\xi}\right)} \frac{\partial P}{\partial \xi} \\
\frac{1}{v^{2}} P_{4}=\left(C_{5}+C_{6}\right) \frac{1}{i \omega\left(i \omega+d_{\eta}\right)} \frac{\partial P}{\partial \eta}
\end{array}\right.
$$

Conduct inverse transformation of Eq.22 to obtain the time domain:

$$
\left\{\begin{array}{l}
\left(\frac{\partial}{\partial t}+d_{\xi}\right)^{2} P_{1}=v^{2}\left(C_{1}^{2}+C_{2}^{2}\right)\left[\frac{\partial^{2} P}{\partial \xi^{2}}-\frac{\partial d_{\xi}}{\partial \xi} \exp \left(-d_{\xi} t\right) * \frac{\partial P}{\partial \xi}\right] \\
\left(\frac{\partial}{\partial t}+d_{\eta}\right)^{2} P_{2}=v^{2}\left(C_{3}^{2}+C_{4}^{2}\right)\left[\frac{\partial^{2} P}{\partial \eta^{2}}-\frac{\partial d_{\eta}}{\partial \eta} \exp \left(-d_{\eta} t\right) * \frac{\partial P}{\partial \eta}\right] \\
\frac{\partial}{\partial t}\left(\frac{\partial}{\partial t}+d_{\xi}\right) P_{3}=v^{2}\left(C_{5}+C_{6}\right) \frac{\partial P}{\partial \xi} \\
\frac{\partial}{\partial t}\left(\frac{\partial}{\partial t}+d_{\eta}\right) P_{4}=v^{2}\left(C_{7}+C_{8}\right) \frac{\partial P}{\partial \eta}
\end{array}\right.
$$

Eq.23 stands for the PML absorbing boundary conditions in the orthogonal curvilinear coordinate system, where * stands for convolution; $d_{\xi}, d_{\eta}$ stand for the attenuation coefficient on the direction of $\xi$ and $\eta$ respectively; $P_{1}, P_{2}, P_{3}, P_{4}$ stand for the intermediate variations introduced.

\section{Numerical examples}

In order to verify the effectiveness of the method, the three speed models were simulated. Model 1 is the homogenous speed model of irregular surface in the sine function (Fig. 3a), the speed of model is $3000 \mathrm{~m} / \mathrm{s}$. Model 2 is the two-layer speed model of irregular surface (Fig. 6a), the speed of the first layer is $2000 \mathrm{~m} / \mathrm{s}$; the speed of the second layer is $3000 \mathrm{~m} / \mathrm{s}$. Model 3 is the 9-layer speed model of irregular surface (Fig. $8 a$ ), the speed from the top to the bottom is $2000 \mathrm{~m} / \mathrm{s}, 2500 \mathrm{~m} / \mathrm{s}, 3000 \mathrm{~m} / \mathrm{s}$, $4000 \mathrm{~m} / \mathrm{s}, 4500 \mathrm{~m} / \mathrm{s}, 5000 \mathrm{~m} / \mathrm{s}, 5500 \mathrm{~m} / \mathrm{s}$ and $6000 \mathrm{~m} / \mathrm{s}$, respectively. The grid spacing of numerical simulation in the calculation domain is $10 \mathrm{~m}$; the shot position is $(1000 \mathrm{~m}, 100 \mathrm{~m})$; the depth of detection point is $20 \mathrm{~m}$ and interval is $10 \mathrm{~m}$. The source function is the Ricker wavelet in time, with a central frequency $f_{0}=30.0 \mathrm{~Hz}$. 
Fig. 3b, Fig. $6 b$ and Fig. 9 stand for the orthogonal body-fitted grid schematic of Model 1, Model 2 and Model 3(Display at every five mesh nodes). It can be seen that our method can achieve a favorable body fitting and orthogonality. Fig. $4 a$ and Fig. $7 a$ is seismic record of Model 1 and Model 2 obtained by the method in this paper. Fig. $4 b$ and Fig. $7 b$ is seismic record of Model 1 and Model 2 obtained through the rectangular mesh finite difference method. From the simulated seismic records, it can be seen that the method in this paper can efficiently eliminate the man-made false scattered wave caused by the ladder-like mesh. From Fig. $4 a$, it can be seen that the direct wave is bended due to the topographical influence. In the sunken region, the travel time is cut off, and the seismic events are not continuous. From Fig. 7a, it can be seen that the reflection events also suffers topographical influence to be distorted and being no longer hyperbola. Fig.5 is snapshots of Model 1 by the method of this paper. Wave field snapshots show that PML absorbing boundary conditions in this paper can effectively eliminate the man-made boundary reflection. Fig. $8 b$ is seismic record of Model 3 obtained through the method in this paper. From Fig. $8 b$, it can be seen that the method can effectively simulate the seismic wave field on any complex irregular surface model.

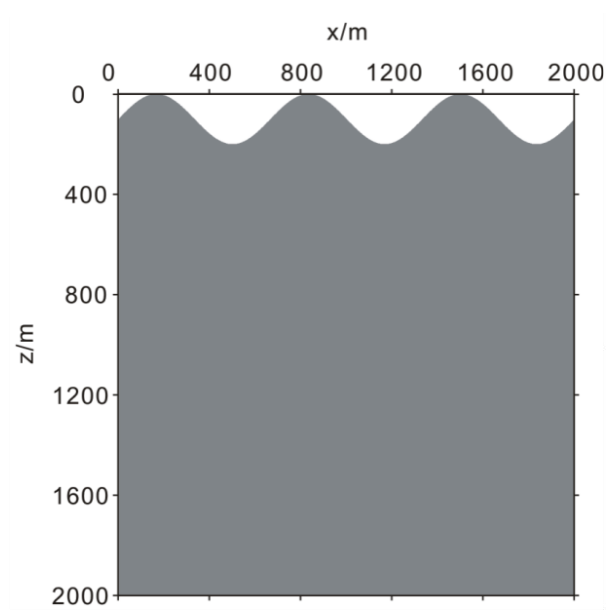

(a)

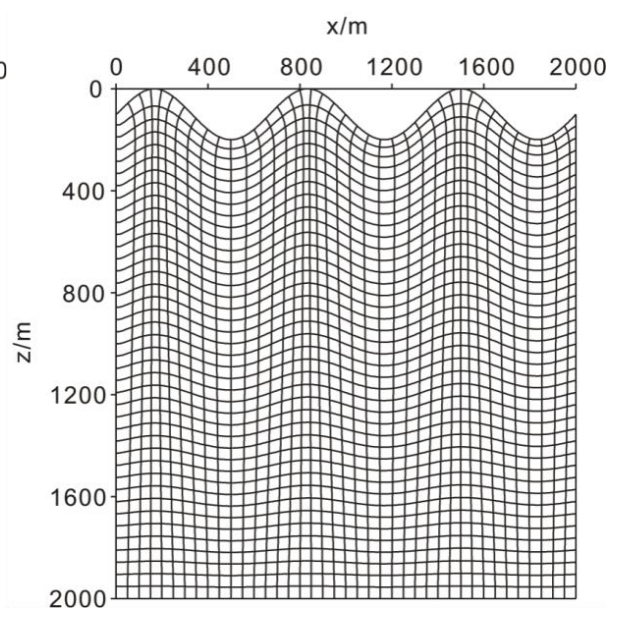

(b)

Figure 3. (a) Velocity model of sine function irregular surface; (b) Orthogonal body-fitted grid subdivision

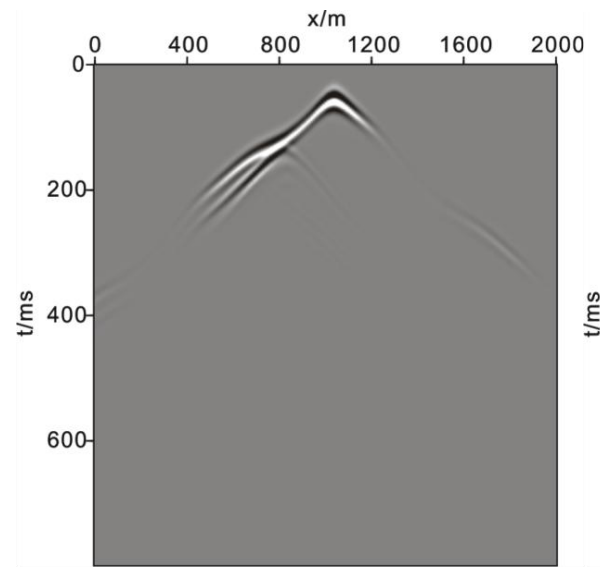

(a)

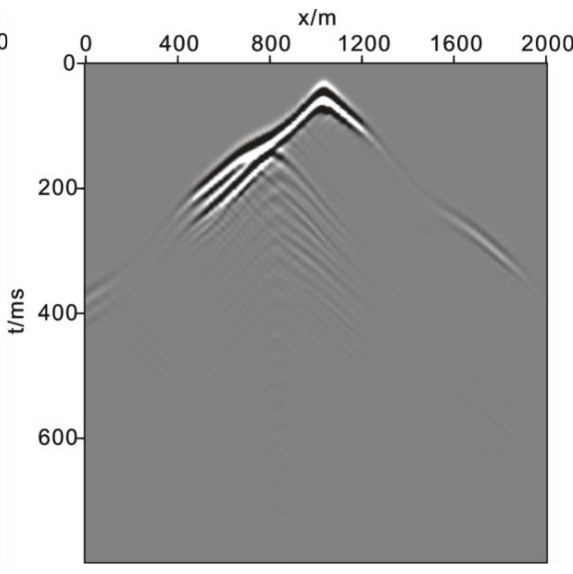

(b)

Figure 4. (a) Wave field snapshot using the method of this paper; (b) Wave field snapshot using rectangular grid finite-difference numerical simulation 


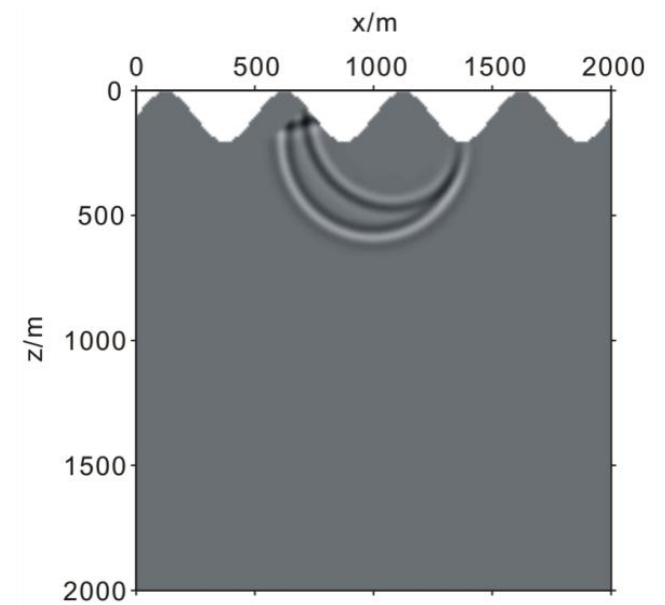

(a) $0.8 \mathrm{~s}$

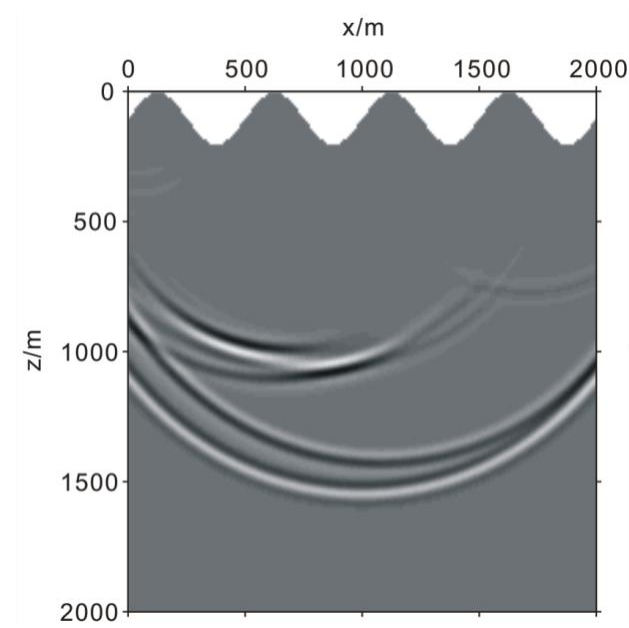

(b) $2.4 \mathrm{~s}$

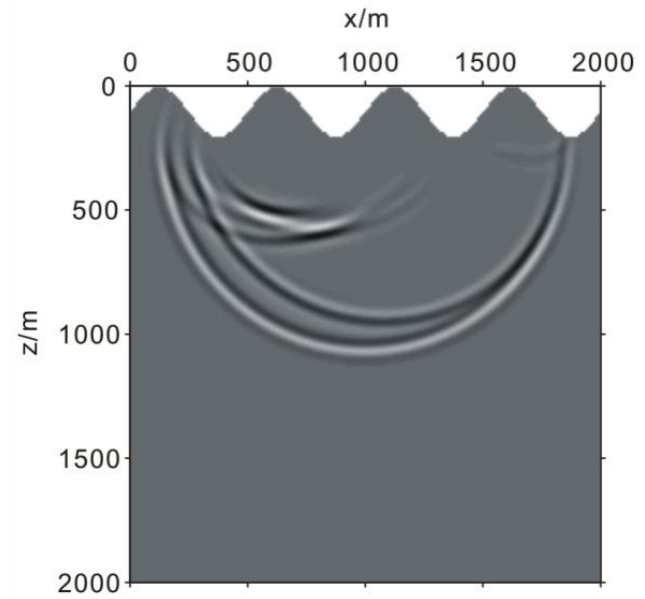

(b) $1.6 \mathrm{~s}$

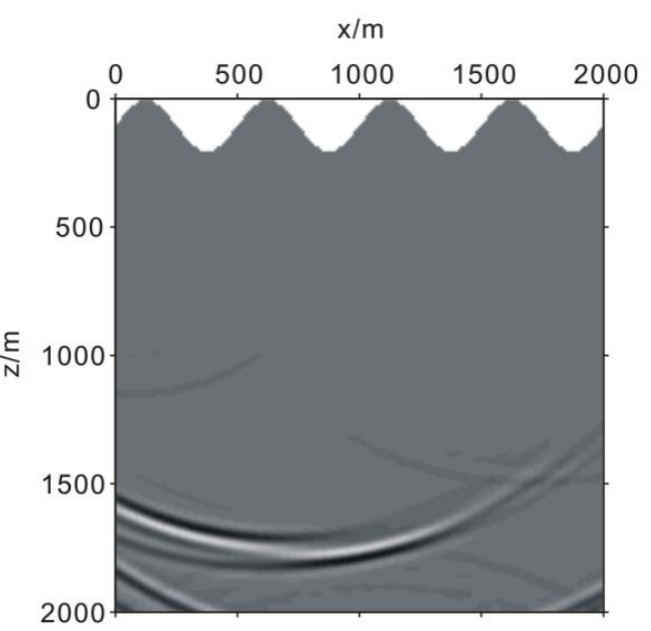

(d) $3.6 \mathrm{~s}$

Figure 5. Wave filed snapshot of model 1

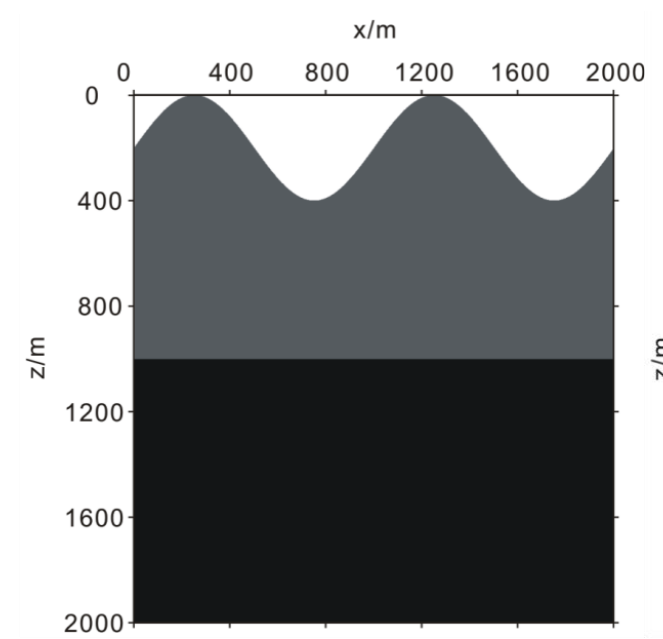

(a)

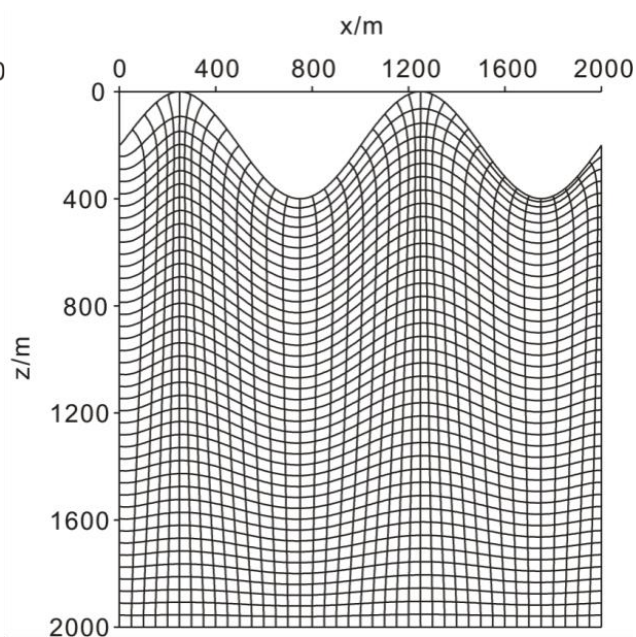

(b)

Figure 6. (a) Two layers velocity model; (b) Orthogonal body-fitted grid subdivision 


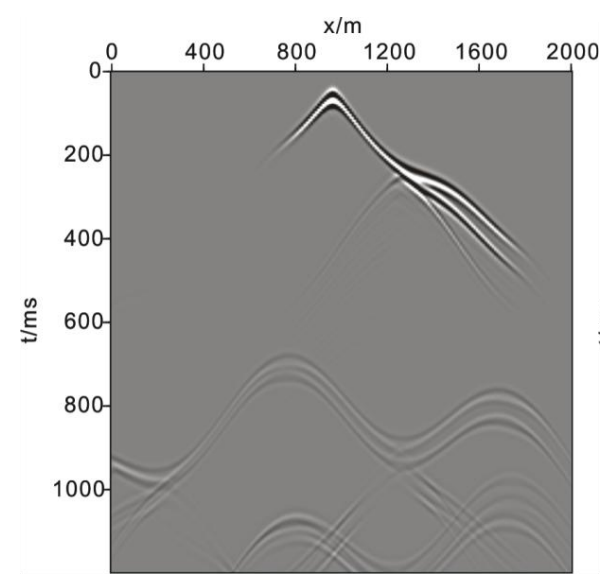

(a)

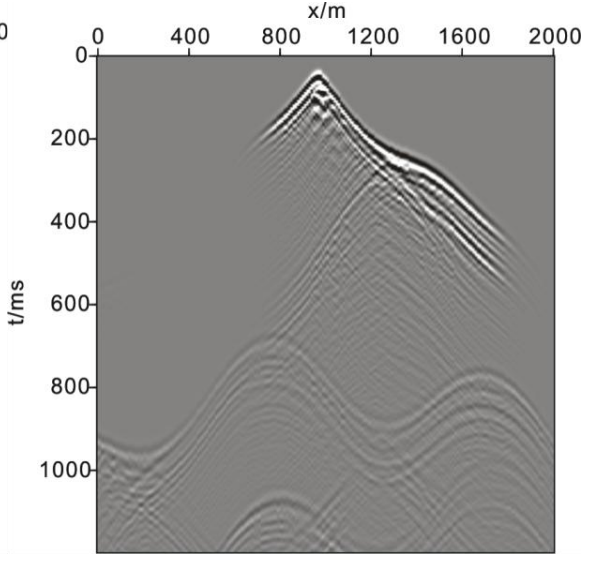

(b)

Figure 7. (a) Seismic record using the method of this paper; (b) Seismic record using rectangular grid finite-difference numerical simulation

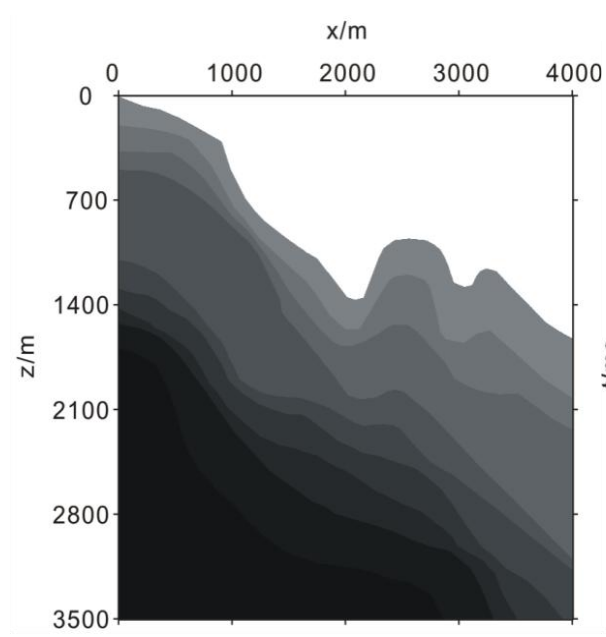

(a)

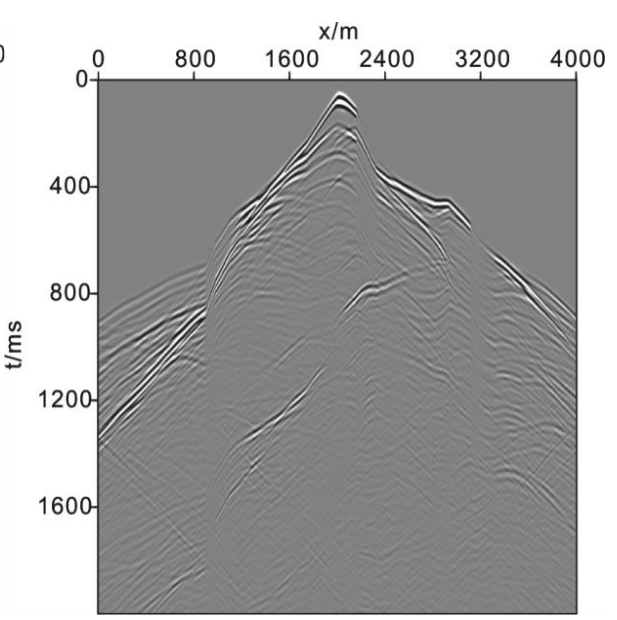

(b)

Figure 8. (a) Complex irregular surface model (b) Seismic record;

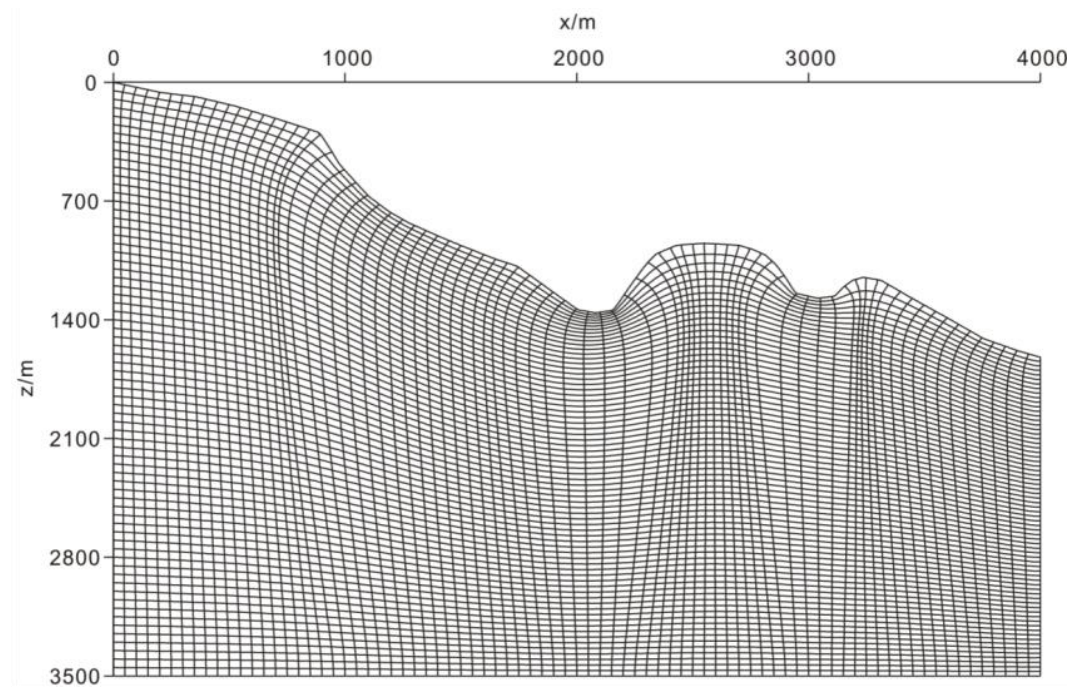

Figure 9. Orthogonal body-fitted grid subdivision of model 3

APPLIED ECOLOGY AND ENVIRONMENTAL RESEARCH 15(3): 465-476. http://www.aloki.hu • ISSN 15891623 (Print) • ISSN 17850037 (Online)

DOI: http://dx.doi.org/10.15666/aeer/1503_465476 (c) 2017, ALÖKI Kft., Budapest, Hungary 


\section{Conclusions}

This paper employs Hilgenstock2D orthogonal body-fitted mesh generation method to discretize the irregular topography models, and simulate the acoustic wave equation under the orthogonal curvilinear coordinate system. Since the body-fitted grid in this paper have a good orthogonality at the boundary, the free boundary conditions can be achieved as Cartesian coordinate system and no need for complex coordinate transformation and interpolation. The application of PML absorbing boundary conditions in the orthogonal curvilinear system can efficiently eliminate the man-made boundary reflection. The numerical examples show that our method have high stability and calculation accuracy, and can efficiently suppress the non-physical scattered waves caused by the ladder-like interface.

Acknowledgments. This study was supported financially by the National Natural Science Foundation of China (41274120, 41404085, 41504084).

\section{REFERENCES}

[1] Berenger, J. P.(1994): A perfectly matched layer for the absorption of electromagnetic waves. - Journal of computational physics 114(2): 185-200.

[2] Chu, C. L., Wang, X. T.(2005): Irregular triangular grid finite-difference modeling of seismic wave. - Journal of Ocean University of China: Natural science edition 35(1): 43-48.

[3] Dong, L. G.(2005): Numerical simulation of seismic wave propagation under complex near surface conditions. - Progress in Exploration Geophysics 28(3): 187-194.

[4] Jastram, C., Behle, A.(1992): Acoustic modelling on a grid of vertically varying spacing. Geophysical prospecting 40(2): 157-169.

[5] Jastram, C., Tessmer, E.(1994): Elastic modelling on a grid with vertically varying spacing1. - Geophysical prospecting 42(4): 357-370.

[6] Hayashi, K., Burns, D.R., Toksöz,M.N.(2001): Discontinuous-grid finite-difference seismic modeling including surface topography. - Bulletin of the Seismological Society of America 91(6): 1750-1764.

[7] Hestholm, S., Ruud, B.(1994): 2D finite-difference elastic wave modelling including surface topography. - Geophysical Prospecting 42(5): 371-390.

[8] Opršal, I., Zahradnik, J.(1999): Elastic finite-difference method for irregular grids. Geophysics 64(1): 240-250.

[9] Sun, J.G.(2007): Methods for numerical modeling of geophysical fields under complex topographical conditions: a critical review. - Global Geology 26(3): 345-362.

[10] Sun, J. G., Jiang, L. L.(2009): Orthogonal curvilinear grid generation technique used for numerical simulation of geophysical fields in undulating surface condition.- Oil Geophysical Prospecting 44(4): 494-500.

[11] Tessmer, E., Kosloff, D., Behle, A.(1992): Elastic wave propagation simulation in the presence of surface topography. - Geophysical Journal International 108(2): 621-632.

[12] Wang, X. C., Liu, X. W.(2007): Simulation and analysis of seismic wavefield on relief surface by 2-D acoustic wave equation. - Oil geophysical prospecting 42(3): 268-276.

[13] Zhang, Z. K., Zhuang, F. G., Zhu, Z. Q.(1997): Two methods of evaluating source terms of elliptic system in the grid generation of nozzle interior field. - Journal of Propulsion Technology 18(2): 95-97. 